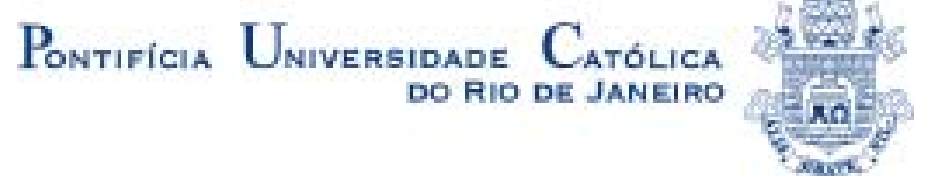

Telma Cristina de Almeida Silva Pereira

O ENSINO DE LÍNGUAS ESTRANGEIRAS COMO UM FATOR DE INCLUSÃO SOCIAL

Tese de Doutorado

Tese apresentada ao Programa de Pós-Graduação em Letras da PUC-Rio como requisito parcial para a obtenção do título de Doutor em Letras

Orientador: Prof. Dr. Jürgen Walter Bernd Heye

Rio de Janeiro, setembro de 2006 
Telma Cristina de Almeida Silva Pereira

\title{
O ENSINO DE LÍNGUAS ESTRANGEIRAS COMO UM FATOR DE INCLUSÃO SOCIAL O desafio da francofonia no Rio de Janeiro
}

Tese apresentada como requisito parcial para obtenção do grau de Doutor pelo Programa de Pós-graduação em Letras da PUC-Rio. Aprovada pela Comissão Examinadora abaixo assinada.

\author{
Prof.Jürgen Walter Bernd Heye \\ Orientador \\ Departamento de Letras - PUC-Rio \\ Prof $^{a}$. Mônica Maria Guimarães Savedra \\ Departamento de Letras - PUC-RIO \\ Prof $^{\mathrm{a}}$. Adriana Ferreira de Sousa de \\ Albuquerque \\ Departamento de Letras - PUC-RIO \\ Prof. Pierre François Georges Guisan \\ Departamento de Letras Neo-Latinas - UFRJ \\ Prof. José Ribamar Bessa Freire \\ Centro de Educação e Humanidades- UERJ \\ Prof. Paulo Fernando Carneiro de Andrade \\ Coordenador Setorial do Centro de Teologia e \\ ciências Humanas - PUC-Rio
}

Rio de Janeiro, 05 de setembro de 2006 
Todos os direitos reservados. É proibida a reprodução total ou parcial do trabalho sem autorização do autor, do orientador e da universidade.

\section{Telma Cristina de Almeida Silva Pereira}

Graduou-se em Letras (português- francês) pela UFRJ (Universidade Federal do Rio de Janeiro) em 1987. Especializou-se em Língua e Literatura Francesa pela UFRJ em 1989. Obteve o título de Mestre em Letras pela UERJ (Universidade do Estado do Rio de Janeiro) em 2002. Participou como membro de projetos de pesquisa na área de sociolingüística apoiados pelo CNPq e pela FAPERJ. Foi bolsista CAPES/PDEE na Université de Provence em 2006. Participou de diversos congressos nacionais e internacionais na área de lingüística e língua francesa. É pesquisadora do grupo de pesquisa Política e Planificação Lingüística no Brasil (CNPq)

FICHA CATALOGRÁFICA

Pereira, Telma Cristina de Almeida Silva

O ensino de línguas estrangeiras como um fator de inclusão social: o desafio da francofonia no Rio de Janeiro / Telma Cristina de Almeida Silva Pereira; orientador: Jürgen Walter Bernd Heye. -2006.

$164 \mathrm{f} . ; 30 \mathrm{~cm}$

Tese (Doutorado em Letras)-Pontifícia Universidade Católica do Rio de Janeiro, Rio de Janeiro, 2006.

Inclui bibliografia

1. Letras - Teses. 2. Política Lingüística. 3. Sociolingüística. 4. Educação. 5. Ensino de Língua Estrangeira. I. Heye, Jürgen Walter Bernd. II. Pontifícia Universidade Católica do Rio de Janeiro. Departamento de Letras. III. Título.

CDD: 400 


\begin{abstract}
A Egberto e Francisca, meus amados e presentes supporteurs,
\end{abstract}

Aos sonhos dos meus pais.

Ao camarada L.J, que aqui e ailleurs, me mostrou que podemos sonhar com os pés no chão. 


\section{Agradecimentos}

Ao querido e grande orientador Jürgen Heye pelo aguçado senso crítico, estímulo e confiança.

A Prof a Mônica Savedra, companheira de projetos, pelo incentivo dado ao tema desta pesquisa que começou a germinar durante o Mestrado, por sua amizade ao longo desse tempo.

Aos demais professores do Programa de Pós- Graduação em Letras da Puc-Rio, em especial a Prof. Rosa Marina, Tânia Saliès e Maria do Carmo cujos ensinamentos contribuíram para o desenvolvimento deste trabalho.

Ao CNPq e à CAPES pelo auxílio financeiro durante o curso.

Aos responsáveis pelas secretarias de Educação dos municípios de Resende, Macaé e Nova Iguaçu, e à Prof. Ana Maria Lucena, responsável pelo setor de línguas estrangeiras da SME do Rio de Janeiro.

À Prof. ${ }^{a}$ Lélia Kalil Thiago, Diretora do DAA/SR-1/UERJ, pela colaboração no levantamento de dados para este trabalho.

Aos funcionários do Programa de Pós-Graduação do Departamento de Letras da PUC-Rio, em especial a Francisca Ferreira Oliveira.

A Université d’Aix-en-Provence, em especial ao Institut de la Francophonie, pela acolhida e apoio durante o estágio Capes/PDEE nesta instituição. 
Em especial, ao Prof. Louis-Jean Calvet, da Université de Provence, sociolingüista sem fronteiras, por sua generosidade, sua contribuição teórica e seu incentivo ao longo do desenvolvimento desta tese.

A Marilúcia Marques, bolsista de IC-PUC-Rio, que me ajudou na aplicação de questionários.

Aos colegas do Programa de Pós-Graduação em Letras, em especial aos que passaram ou que passam pela área de sociolingüística: Kelly, Karina, Carmem,Tânia, Beate... 


\section{Resumo}

Pereira, Telma Cristina de Almeida Silva. Heye, Jürgen Walter Bernd (Orientador). $O$ ensino de línguas estrangeiras como um fator de inclusão social: o desfio da francofonia no Rio de Janeiro. Rio de Janeiro, 2006. 164p. Tese de Doutorado - Departamento de Letras, Pontifícia Universidade Católica do Rio de Janeiro.

A pesquisa $\mathrm{O}$ ensino de línguas estrangeiras como um fator de inclusão social: o desafio da francofonia no Rio de Janeiro tem por objetivo analisar o efeito das políticas lingüísticas para o ensino de línguas estrangeiras no Estado do Rio de Janeiro, partindo de um pressuposto básico da sociolingüística que é aquele que propõe estabelecer uma relação entre língua e sociedade. Esta análise considera dados provenientes do contexto histórico, econômico e cultural de alguns municípios do Estado selecionados para este estudo. Consideramos a inserção sócio-econômica dos cidadãos nas regiões estudadas como um dos critérios para julgar a eficácia das políticas lingüística adotadas. Para tanto, consideramos as políticas lingüísticas no domínio das línguas estrangeiras levando primeiramente em conta as medidas legalmente explicitadas através de leis e decretos, assim como procuramos mostrar de que modo uma parcela da sociedade resolve as lacunas relacionadas à oferta de ensino de línguas no sistema educacional.

\section{Palavras-chave}

Política Lingüística, Sociolingüística, Ensino de Línguas Estrangeiras. 


\section{Abstract}

Pereira, Telma Cristina de Almeida Silva. Heye, Jürgen Walter Bernd (Advisor) The enseigment of the foreign languages as a factor of social inclusion: the challenge of the francophonie in Rio de Janeiro. Rio de Janeiro, 2006. 164p. Phd. Thesis - Departamento de Letras, Pontifícia Universidade Católica do Rio de Janeiro

This study analyzes the effect of language policies for the teaching of foreign languages in the State of Rio de Janeiro, Brazil, based on the basic postulate of sociolinguistics - that of establishing a relation between language and society. Our analysis takes into account a set of data derived from the historical, economic and social context of a few selected municipalities of the State. We consider the socioeconomic insertion of citizens in the regions under investigation as one of the criteria to evaluate the efficiency of the language policies adopted. Thus, we consider the language policies as reflected in the teaching of foreign languages, taking into account, first of all, the legal means and measures, as expressed in laws and decrees. We also attempt to show how a part of the society fills the gaps related to foreign language teaching-or the lack thereof- in the educational system.

\section{Keywords}

Sociolinguistics, Language policies, Foreign Language, Teaching 


\section{Resumen}

Pereira, Telma Cristina de Almeida Silva. Heye, Jürgen Walter Bernd (Director). El enseigment de las lenguas extrajeras como un factor de inclusión social: lo desafío de la francofonía en Río de Janeiro. Rio de Janeiro, 2006. 164p. Tesis de Doctorado - Departamento de Letras, Pontifícia Universidade Católica do Rio de Janeiro.

Este trabajo tiene como objetivo analizar el efecto de las políticas lingüísticas para enseñar lenguas extranjeras en el Estado de Rio de Janeiro, partiendo del principio básico de la sociolingüística que es aquel que propone establecer una relación entre lengua y sociedad. Este análisis considera datos provenientes del contexto histórico, económico y cultural de algunos municipios del Estado. Consideramos la inserción socio-económica de los ciudadanos en las regiones estudiadas como uno de los criterios para juzgar la eficacia de las políticas lingüísticas adoptadas. Para tal efecto, consideramos las medidas legalmente explicitadas a través de leyes y decretos y posteriormente, si tales políticas corresponden a los perfiles de los municipios.

\section{Palabras-clave}

Sociolingüística, políticas lingüística, lengua extranjeras 


\section{Zusammennfassung}

Pereira, Telma Cristina de Almeida Silva. Heye, Jürgen Walter Bernd (Direktor). Der enseigment der Fremdsprachen wie ein Faktor sozialer Einschließung: der defis der Frankophonie in Rio de Janeiro. Rio de Janeiro, 2006. 164p. Doktorarbeit - Departamento de Letras, Pontifícia Universidade Católica do Rio de Janeiro.

Ziel der vorliegenden Arbeit ist es, ausgehend von der grundlegenden Annahme der Soziolinguistik, die eine Beziehung zwischen Sprache und Gesellschaft herstellt, die Auswirkungen der sprachpolitischen Maßnahmen auf den Fremdsprachenunterricht im Bundesstaat von Rio de Janeiro zu analysieren. Die vorliegende Analyse stützt sich auf Daten des historischen, wirtschaftlichen und kulturellen Kontexts einiger Regierungsbezirke des Bundesstaats, die für diese Studie ausgewählt wurden. Die sozioökonomische Integration der Bürger in die untersuchten Regionen wird als eines der Kriterien benutzt, um die Effizienz der sprachpolitischen Maßnahmen zu beurteilen. Dafür wird die Sprachpolitik bezüglich der Fremdsprachenbeherrschung berücksichtigt, v.a. solche Maßnahmen, die rechtsgültig durch Gesetze und Dekrete ausgedrückt werden und darauf folgend wird untersucht, ob diese den Anforderungen der Bezirkskreise entsprechen.

\section{Schlüsselwörter}

Soziolinguistik, Sprachpolitik, Erziehung. 


\section{Résumé}

Pereira, Telma Cristina de Almeida Silva. Heye, Jürgen Walter Bernd (Directeur de thèse). L'enseigment des langues étrangères comme um facteur d'inclusion sociale: le defi de la francophonie à Rio de Janeiro. Rio de Janeiro, 2006. 164p. Thèse de Doctorat - Departamento de Letras, Pontifícia Universidade Católica do Rio de Janeiro.

Ce travail a pour but d'analyser l'effet des politiques linguistiques sur l'enseignement des langues étrangères à l'état de Rio de Janeiro, prenant par principe l'un des concepts de bases de la sociolinguitsique qui propose d'établir un lien entre langue et société. Cette analyse prend en compte des données issues du contexte historique, économique et culturel de quelques municipalités de l'État. Nous avons consideré l'insertion sócio-économique des citoyens des régions étudiées comme l'un des critères d'évaluation de l'efficacité des politiques linguistiques adoptées. Notre point de départ ont été les politiques des langues étrangères explicitées à travers des lois et des décrets pour ensuite vérifier comment la société agit face aux lacunes concernant l'offre de l'enseignement des langues dans le système éducationnel.

\section{Mots-clefs}

Politique linguistique, sociolinguistique, éducation. 


\section{SUMÁRIO}

1. Introdução 21

1.1 Delimitação do tema 25

1.2. Justificativa da pesquisa 28

$\begin{array}{ll}1.3 \text { Objetivos da pesquisa } & 29\end{array}$

1.4. Metodologia adotada 30

1.5 Organização do trabalho 30

2. Política lingüística: fundamentação teórica 31

2.1 Introdução 31

2.2 Política lingüística: conceitos $\quad 34$

2.3 Intervenções in vivo e in vitro: uma relação de forças 41

2.4 Intervenções lingüísticas no Brasil: entre o in vivo e o in vitro 43

3. O ensino de línguas estrangeira no Brasi

e a Lei de Diretrizes e Bases 49

3.1 Introdução $\quad 49$

3.2 As primeiras Intervenções lingüísticas no Brasil 51

3.3 Percurso histórico da LDB 55

$3.4 \mathrm{O}$ ensino de línguas estrangeiras segundo a LDB 67

4. Globalização e política lingüística para o ensino de línguas estrangeiras $\quad 74$

4.1 Introdução $\quad 74$

$\begin{array}{ll}4.2 \text { Globalização: conceitos } & 77\end{array}$

4.3 Línguas e colonização: "La compãnera del Império" 81 
4.4 Línguas e globalização: "a língua é minha Pátria, e eu não tenho Pátria, tenho mátria, e quero frátria..." 86

4.5 As escolas bilíngües no Rio de Janeiro 89

4.6 Ensino de línguas estrangeiras e escolas bilíngües:

entre o bilingüismo horizontal e o bilingüismo vertical 92

4.7 A escola bilíngüe francesa (Lycée Molière): um exemplo

de bilingüismo vertical

95

5. O descompasso entre a política lingüística oficial

e a realidade dos municípios fluminenses:Rio de Janeiro,

Macaé, Resende 100

5.1 Introdução 100

5.2 Origem dos municípios como unidades administrativas 101

5.3 O Estado do Rio de Janeiro 105

5.4 Divisão regional do estado do Rio de Janeiro 110

5.5 Rio de Janeiro: "cidade maravilha purgatório

da beleza e do caos" 113

5.5.1 Ensino de línguas estrangeiras no município do Rio de Janeiro 115

5.6 Resende: pólo do desenvolvimento do Médio Paraíba 120

5.7 Macaé: a capital da indústria do petróleo 123

5.7.1 Ensino de línguas em Macaé 125

6. O reflexo das políticas de ensino de línguas

estrangeiras no Rio de Janeiro 127

6.1 Introdução $\quad 127$

6.2 Línguas estrangeiras no vestibular estadual 128

6.3 Formação e representação lingüística 132 
7. A francofonia: descentralizar para democratizar

o ensino do francês 140

7.1 Introdução 140

7.2 A francofonia: origens $\quad 141$

7.3 Áreas de atuação da francofonia $\quad 145$

7.4 A descentralizacão do ensino do francês:

um desafio para a francofonia 146

8.Considerações finais 148

9. Referências bibliográficas 154 


\section{Lista de Tabelas}

Tabela 1: Perfil das escolas bilíngües no município do Rio de Janeiro

Tabela 2: Línguas estrangeiras nas escolas vinculadas

à AEFE localizadas os países membros do Mercosul

99

Tabela 3: Empresas francesas no Estado do Rio de Janeiro

Tabela 4: Número de escolas em 2004

Tabela 5: Cursos de línguas no Estado do Rio de Janeiro

Tabela 6: Números relativos ao vestibular Uerj 2006,

$2^{\mathrm{a}}$ fase - exame discursivo

Tabela 7: Que língua(s) você fala

Tabela 8: A língua mais útil

Tabela 9: A língua mais fácil

Tabela 10: A língua mais difícil

Tabela 11: A língua que gostaria que os filhos apreendessem

Tabela 12: A língua mais bonita

Tabela 13: Regiões ou cidades plurilíngües no Brasil 


\section{Lista de Figuras}

Figura1: Mapa do estado do Rio de Janeiro mostrando as principais cidades das regiões de governo.

Em destaque, as cidades abordadas neste estudo 106

Figura 2: Regiões de Governo do Estado do Rio de Janeiro

Figura 3 - Localização das filiais da Aliança Francesa

nas regiões com maiores índices de desenvolvimento

humano (IDH) da cidade do Rio de Janeiro

Figura 4 - Distribuição do Produto Interno Bruto

no Estado do Rio de Janeiro 


\section{Lista de Quadros}

Quadro1: Dados socioeconômicos do Estado do Rio de Janeiro

105

Quadro 2: Opção língua estrangeira na primeira

fase do vestibular estadual 2006

131

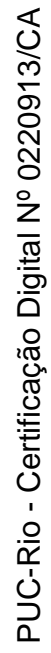




\section{Siglas}

ABM: Academia de Bombeiros Militar D.Pedro II

APM: Academia de Polícia Militar D.João VI

ABRALIN: Associação Brasileira de Lingüística

AUF: Agence Universitaire de la Francophonie

CAPES: Coordenação de Aperfeiçoamento de Pessoal de Nível Superior

CIDE: Centro de Informações e Dados do Rio de Janeiro.

CIPLA: Congresso Internacional de Política Lingüística na América do Sul.

CNPq: Conselho Nacional de Desenvolvimento Científico e Tecnológico

CPLP: Comunidade dos Países de Língua Portuguesa

ENPLE: Encontro Nacional sobre Política de Ensino de Línguas Estrangeiras.

FILE: Fórum Internacional de Ensino de Línguas Estrangeiras.

GELNE: Grupo de Estudos Lingüísticos do Nordeste

IBGE: Instituto Brasileiro de Geografia e Estatística

INEP: Instituto Nacional de Estudos e Pesquisas Educacionais Anísio Teixeira. 
IPEA: Instituto de Política Econômica e Aplicada

IPOL: Instituto de Investigação e Desenvolvimento em Política Lingüística.

LDB: Lei de Diretrizes e Base da Educação Nacional

LE: língua estrangeira

MERCOSUL: Mercado Comum do Sul

OEI: Organização de Estados Ibero-Americanos.

OIF: Organização Intergovernamental da Francofonia

PCNs: Parâmetros Curriculares Nacionais.

PNE: Plano Nacional de Educação

SBPC: Sociedade Brasileira para o Progresso da Ciência.

UENF: Universidade Estadual do Norte Fluminense Darcy Ribeiro 